\title{
hTERT expression in melanocytic lesions: an immunohistochemical study on paraffin-embedded tissue
}

Background: Telomerase plays a role in the immortalization of cells and carcinogenesis. Previous studies have yielded conflicting results on whether human telomerase RNA (hTER) expression differs in nevi, atypical nevi and melanomas using polymerase chain reaction-based telomeric repeat amplification protocol or in situ hybridization assays. The aim of this study was to evaluate human telomerase reverse transcriptase (hTERT) staining in melanocytic lesions on paraffinembedded tissues.

Methods: Paraffin-embedded sections from 12 acquired nevi, seven dysplastic nevi, 11 Spitz nevi, eight primary invasive melanomas, and three metastatic melanomas were studied for staining intensity $(0-3+)$ and percentage of labeled cells with anti-hTERT.

Results: hTERT staining was observed in most cells $(>75 \%)$, in all but three lesions, and was of greater intensity in the nucleus, especially the nucleolus, compared with the cytoplasm. Spitz nevi tended to have weaker hTERT staining $($ mean $=1.7)$ compared with acquired nevi $($ mean $=2.2)$, dysplastic nevi $($ mean $=2.4)$, primary melanomas $($ mean $=2.4)$, or metastatic melanomas $($ mean $=3)$.

Conclusions: Although telomerase activity was weaker in Spitz nevi, there was overlap with other nevi and primary invasive melanomas in our small series. Thus, hTERT expression does not appear to be a reliable adjunct to the histological diagnosis of primary melanocytic lesions.

Fullen DR, Zhu W, Thomas D, Su LD. hTERT expression in melanocytic lesions: an immunohistochemical study on paraffin-embedded tissue.

J Cutan Pathol 2005: 32: 680-684. (C) Blackwell Munksgaard, 2005.

\section{Douglas R. Fullen ${ }^{1,2}$, Weijian Zhu $^{1}$, Dafydd Thomas ${ }^{1,3}$ and Lyndon D. Su ${ }^{1,2}$}

${ }^{1}$ Department of Pathology, ${ }^{2}$ Department of Dermatology, and ${ }^{3}$ Department of Internal Medicine, University of Michigan Medical Center, Ann Arbor, MI, USA

Douglas R. Fullen, MD, M3261, Medical Sciences I, Department of Pathology, University of Michigan Medical Center, 1301 Catherine, Ann Arbor, MI 48109-0602, USA

Tel: +17347644460

Fax: +17347644960

e-mail: dfullen@umich.edu

Accepted for Publication March 14, 2005
Telomeres are composed of tandem nucleotide repeats (TTAGGG) $n$ and are located on the termini of chromosomes in all vertebrates. The role of telomeres is to protect chromosomes from degradation and aberrant recombination during replication. ${ }^{1-5}$ Telomerase is a ribonucleoprotein DNA polymerase that is responsible for maintaining the length of telomeres on the end of chromosomes. ${ }^{6,7}$ This enzyme is repressed in human somatic cells, with a few exceptions, resulting in progressive loss of telomeres and shortening of the chromosome with successive cell divisions. Eventually, chromosomes reach a critical length at which cell division ceases, senescence begins, and the cell ultimately undergoes apoptosis or cell death. ${ }^{8,9}$ In contrast, telomerase activity is detectable in germline cells, somatic cells during fetal development, germinal centers of lymph nodes, regenerative epithelium of the gastrointestinal tract, proliferative endometrium, the bulge region of hair follicles, and stem cells of the epidermis. $10-15$

Immortalized cell lines of a vast array of human cancers, such as breast, colorectal and ovarian cancer, and melanoma, for example, have been shown 


\section{hTERT immunostain in melanocytic lesions}

to possess telomerase activity. ${ }^{16-22}$ Additional cutaneous neoplasms that have been shown to express telomerase activity include basal cell carcinoma, in situ and invasive squamous cell carcinoma, eccrine carcinoma, poroma and porocarcinoma, trichilemmal carcinoma, and seborrheic keratosis. ${ }^{23-25}$ Thus, telomerase appears to play a role in the unregulated proliferation and ultimate immortality of somatic cells. $^{26-30}$

Previous studies evaluating human telomerase RNA (hTER) expression in melanocytic lesions of the skin, including various types of nevi and melanomas, using polymerase chain reaction (PCR)based telomeric repeat amplification protocol (TRAP) or in situ hybridization assays have yielded conflicting results. Rudolph et al. ${ }^{31}$ demonstrated distinct differences in telomerase activity between nevi and melanomas, but not between common nevi and dysplastic nevi, by TRAP assay, and that levels correlated with tumor progression in melanomas. Ramirez et al. ${ }^{32}$ also found that telomerase RNA levels increased from benign to malignant melanocytic lesions and from primary to metastatic melanomas by TRAP assay and confirmed the enzyme expression in tumor cells by in situ hybridization on paraffin-embedded tissue sections. In contrast, Guttman-Yassky et al. ${ }^{33}$ reported similar moderate-to-high intensity RNA levels in Spitz nevi, ordinary nevi, and melanomas by in situ hybridization for hTER.

To our knowledge, telomerase activity has not been evaluated on paraffin-embedded tissue sections in melanocytic lesions. The goal of this study was to evaluate a spectrum of melanocytic lesions, including common acquired nevi, Spitz nevi, atypical (dysplastic) nevi, primary invasive melanomas, and metastatic melanomas, to determine whether differences exist in the intensity or pattern of human telomerase reverse transcriptase (hTERT) expression between these lesions and whether this antibody has diagnostic utility in their evaluation.

\section{Materials and methods}

Formalin-fixed, paraffin-embedded archival tissue blocks from 30 benign melanocytic lesions (12 common acquired nevi, seven dysplastic nevi, and 11 Spitz nevi), and 11 melanomas (8 primary invasive and 3 metastatic) were retrieved following a SNOMED search of our laboratory data system. The diagnoses were rendered by one of three staff dermatopathologists on the Dermatopathology Service at the University of Michigan.

Immunohistochemical staining for hTERT was performed on all 41 melanocytic lesions. Briefly, $4-\mu \mathrm{m}$ thick sections were cut and placed on charged slides. The slides were deparaffinized, rehydrated, and endogenous peroxidases were blocked by incubation with $3 \% \mathrm{H}_{2} \mathrm{O}_{2}$. Antigen retrieval was accomplished by incubating the slides with $10-\mathrm{mM}$ citrate buffer at $\mathrm{pH} 6.0$ and microwaving for 20 min. The slides were stained with a primary monoclonal antibody to hTERT (1:50; Novocastra, Newcastle, UK). The primary antibody titer used in this study was selected based on the dilution that produced an optimum staining pattern as judged in titration studies performed on tonsil and melanoma tissue microarray controls and was in concordance with the manufacturer's recommendation. Immunohistochemical staining was performed on a DAKO Autostainer (DAKO, Carpinteria, CA) using DAKO LSAB+ and 3,3'-diaminobenzidine as the chromogen. The slides were counterstained with Mayer's hematoxylin. Positive and negative control slides were performed. The extent of staining, as determined by the percentage of lesional cells $(0 /-, \quad 1-25 \% / 1+, 26-75 \% / 2+$, and $>75 \% / 3+)$ and the intensity (negative/ 0 , weak/1+, moderate $/ 2+$, and strong/3+), was independently evaluated by three observers, including two board certified dermatopathologists (LDS and DRF) and were recorded semiquantitatively and with good concordance.

The Institutional Review Board of the University of Michigan approved this study.

\section{Results}

The percentage of lesional cells expressing hTERT and the intensity of staining are presented in Tables 1 and 2, respectively. hTERT staining was identified in all 41 melanocytic lesions. The vast majority $(>75 \%)$ of lesional cells expressed hTERT in the melanocytic lesions, with the exception of one acquired nevus, one Spitz nevus, and one dysplastic nevus, which showed $25-75 \%$ of lesional cells staining. The pattern of hTERT expression was uniform throughout the lesion, with approximately the same degree of staining in the lower half as the upper half of the dermal component in benign melanocytic nevi and melanomas. The nucleus, especially the nucleolus, showed greater staining intensity compared with the cytoplasm of the melanocytes.

The staining intensity varied between the different types of melanocytic lesions. In general, the least amount of hTERT staining was seen in Spitz nevi (mean $=1.7)$. In fact, weak $(1+)$ labeling for hTERT was observed in five of 11 Spitz nevi (Fig. 1A), whereas six of 11 Spitz nevi showed moderate $(2+)$ or strong $(3+)$ staining. Common acquired nevi that were either compound or intradermal showed greater staining $($ mean $=2.2)$ than 


\section{Fullen et al.}

Table 1. The intensity of hTERT protein expression in skin biopsies of melanocytic lesions

\begin{tabular}{lllll}
\hline & $1+$ & $2+$ & $3+$ & Mean \\
\hline Acquired nevi & 2 & 6 & 4 & 2.2 \\
Spitz nevi & 5 & 4 & 2 & 1.7 \\
Dysplastic nevi & 0 & 4 & 3 & 2.4 \\
Melanoma & 1 & 3 & 4 & 2.4 \\
Metastatic melanoma & 0 & 0 & 3 & 3.0 \\
\hline
\end{tabular}

$1+=$ weak; $2+=$ moderate; $3+=$ strong.

Spitz nevi, with only two of 12 examples possessing weak $(1+)$ hTERT staining and 10 of 12 examples having moderate $(2+)$ or strong $(3+)$ staining (Fig. 1B). Dysplastic nevi and primary invasive melanomas had the same average $($ mean $=2.4$ ) for hTERT staining, although one invasive melanoma had weak $(1+)$ staining in contrast to no dysplastic nevi. On the other hand, a slightly higher percentage of invasive melanomas $(4 / 8,50 \%)$ than dysplastic nevi $(3 / 7,43 \%)$ had strong (3+) labeling (Fig. 1C,D). Metastatic melanomas had the most hTERT expression $($ mean $=3)$, with strong $(3+)$ expression in all three cases (Fig. 1E).

In the skin adjacent to the melanocytic lesions, primarily nuclear staining for hTERT was observed in all levels of the epidermis, hair follicle epithelium, in particular the outer few layers along the entire length of the follicle and diffusely in hair bulbs, sebaceous lobules, sweat duct and glandular epithelium, and vascular endothelium to at least a moderate $(2+)$ degree (Fig. 1F). Positive and negative control slides were appropriate.

\section{Discussion}

Our study has proven that hTERT can be reliably detected in paraffin-embedded tissue sections in a variety of melanocytic lesions that includes both nevi (common acquired and Spitz) and melanomas. This method is very useful because, in contrast to the PCR-based TRAP assay employed in some of the previous studies, the staining can be visualized in the cells of interest. Moreover, it is clear that many normal tissue structures in skin reproducibly express

Table 2. The extent of hTERT protein expression in skin biopsies of melanocytic lesions

\begin{tabular}{lllll}
\hline & $1+$ & $2+$ & $3+$ & Mean \\
\hline Acquired nevi & 0 & 1 & 11 & 2.9 \\
Spitz nevi & 0 & 1 & 10 & 2.9 \\
Dysplastic nevi & 0 & 1 & 6 & 2.9 \\
Melanoma & 0 & 0 & 8 & 3.0 \\
Metastatic melanoma & 0 & 0 & 3 & 3.0 \\
\hline
\end{tabular}

$1+=1-25 \%$ of lesional cells; $2+=26-75 \%$ of lesional cells; $3+\geq 75 \%$ of lesional cells. telomerase. In agreement with the observations of Guttman-Yassky et al., we observed telomerase expression in keratinocytes at all levels of the epidermis and within sebaceous glands, hair follicles, and sweat glands, although they employed in situ hybridization for hTER expression, and we evaluated hTERT expression by immunohistochemistry. We are unaware of any immunohistochemical studies, to date, that have evaluated hTERT staining on skin lesions. It is not surprising that hTERT is seen predominantly in the nucleolus of the nucleus, considering that the target of the antibody is a ribonucleoprotein DNA polymerase.

In this series of 41 melanocytic lesions, we were able to demonstrate hTERT staining in all lesions. A trend of increasing mean hTERT expression from nevi to dysplastic nevi and primary invasive melanomas to metastatic melanomas was observed. This finding suggests a role for telomerase in tumor progression, which is not surprising based on previously published data. For instance, Ramirez et al. ${ }^{32}$ demonstrated increased mean levels of telomerase activity by the PGR-based TRAP assay for subcutaneous and nodal metastases of melanoma when compared with primary invasive melanomas and also with increasing Clark's level for the primary invasive melanomas. Subsequently, Glaessl et al. ${ }^{34}$ reported significant differences in percentage of cases with positive telomerase levels by a PCRbased TRAP ELISA assay for metastatic melanomas (92\%), primary invasive melanomas (90\%), and atypical nevi (80\%) compared with common nevi $(28 \%)$ and normal skin (12\%). Mirraco et al. also demonstrated increasing mean telomerase activity using a PCR-based TRAP assay from benign nevi to dysplastic nevi to invasive melanomas to metastatic melanomas. ${ }^{35}$ Finally, Rudolph and colleagues $^{31}$ showed similar telomerase activity between common and dysplastic nevi, but the levels were much lower than for melanomas, although they noted that all three groups had overlapping ranges. Our findings are essentially in agreement with these observers, although, in comparison with Rudolph et al. our dysplastic nevus group showed similar mean telomerase expression to primary invasive melanomas and slightly greater than common acquired nevi. In contrast to Guttman-Yassky et al. who showed moderate-to-high hTER expression in Spitz nevi, ordinary nevi, and melanomas, ${ }^{33}$ we found the lowest semiquantitative hTERT staining in our Spitz nevus group. Our finding of the lowest mean telomerase activity in the Spitz nevus group is similar to Tosi and colleagues. ${ }^{36}$ However, their Spitz nevus group did not have an overlapping range of mean relative telomerase activity compared with their melanoma groups by TRAP assay, whereas our Spitz nevus group did overlap with 
Fig. 1. Human telomerase reverse transcriptase (hTERT) protein is expressed in skin biopsies of melanocytic lesions. (A) Spitz nevus with $1+$ nuclear staining. (B) Intradermal nevus with $3+$ nuclear staining. (C) Dysplastic compound nevus with 3+ nuclear staining. (D) Primary invasive melanoma with 3+ nuclear staining. (E) Dermal satellite metastasis of melanoma with $3+$ nuclear staining. (F) Keratinocyte nuclei of the epidermis stain positively for hTERT in this compound nevus.
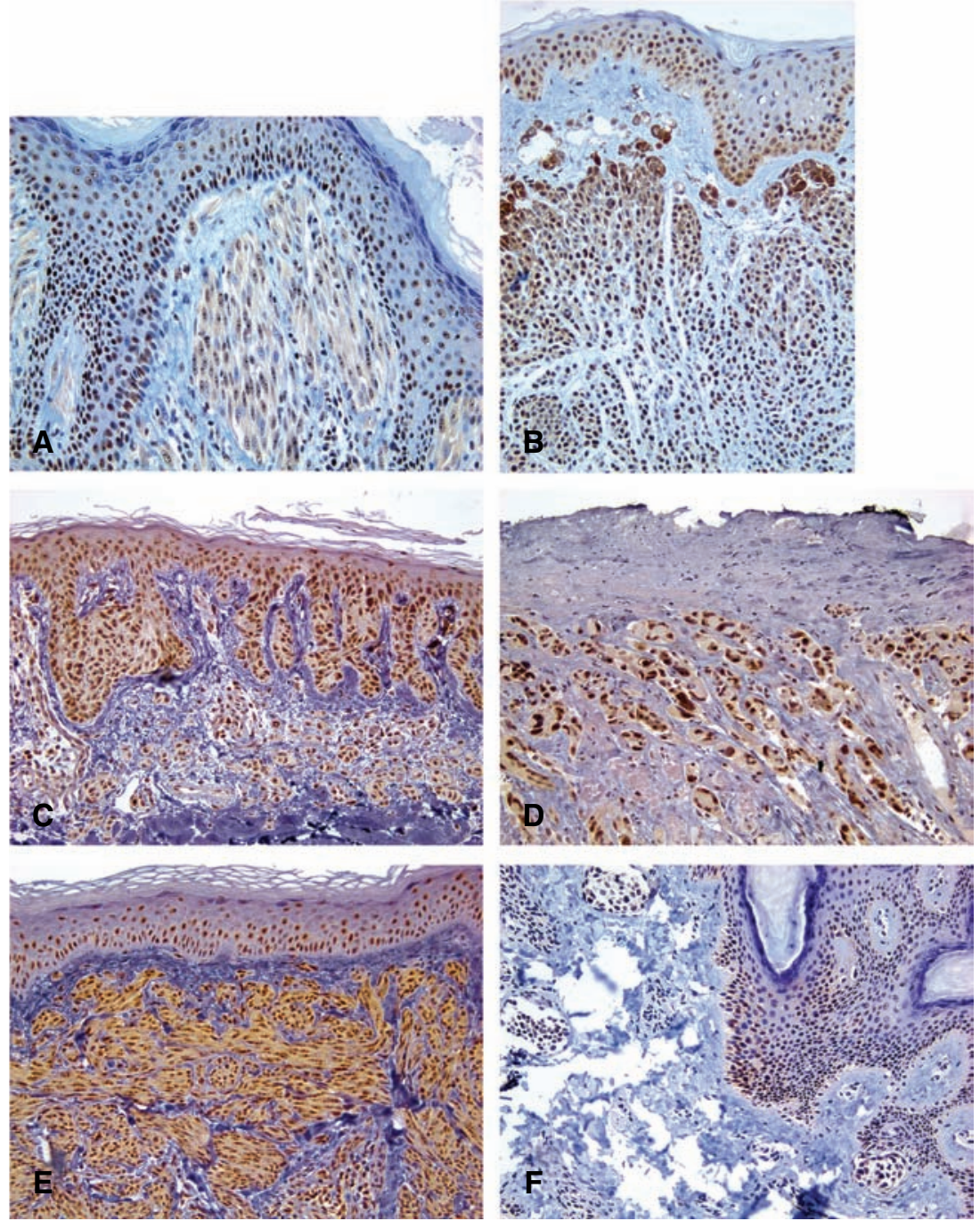

our melanoma group with respect to immunostain intensity for hTERT.

On the basis of significant differences in telomerase activity between nevi and melanomas in some studies and the recent availability of a primary antibody to hTERT for immunohistochemical staining on paraffin-embedded sections, we were interested in evaluating the staining pattern and intensity of hTERT in a spectrum of melanocytic lesions to determine whether this antibody had diagnostic utility. With the exception of metastatic melanomas, which showed uniformly strong hTERT staining in all cases, there was considerable overlap in staining intensity among the remaining groups, including with the metastatic melanoma group in this study. Thus, hTERT expression, unfortunately, is not a reliable discriminator between benign and malignant melanocytic lesions in paraffin-embedded tissues and, therefore, does not appear to have a role as an adjunct to the histological differential diagnosis of melanocytic lesions. However, it is possible that this antibody may prove useful as a prognostic marker for melanoma or specific subtypes or to identify lesions that would be amenable to specific therapy that would inhibit telomerase. The difficulty in the latter instance is that normal skin structures have telomerase activity, suggesting that there is potential for untoward side effects unless telomerase activity is coupled to a melanoma-specific target mechanism.

In summary, hTERT staining can be detected in a broad spectrum of melanocytic lesions, as well as some normal skin structures. A trend of increasing mean hTERT staining from benign nevi to atypical nevi and invasive melanoma to metastatic melanoma suggests a role in tumor progression, in keeping with previous observations. Finally, this antibody does not appear to have a role in diagnostic pathology for the determination between a benign and malignant melanocytic lesion for a given case, within the limitations of the specific types of melanocytic lesions studied in this series. The potential role of immunohistochemical staining for hTERT in other cutaneous neoplasms remains to be elucidated. 


\section{Fullen et al.}

\section{Acknowledgements}

These findings were presented in abstract form at the 2004 Annual Meeting of the American Society of Dermatopathology. Dr Dafydd Thomas is a Walther Cancer Research Institute Scholar and thanks them for their support.

\section{References}

1. Blackburn EH. Structure and function of telomeres. Nature 1991; 350: 569.

2. Greider CW. Mammalian telomere dynamics: healing, fragmentation shortening and stabilization. Curr Opin Genet Dev 1994; 4: 203.

3. Harley CB, Futcher AB, Greider CW. Telomeres shorten during ageing of human fibroblasts. Nature 1990; 345: 458.

4. Allsopp RC, Vaziri H, Patterson C et al. Telomere length predicts replicative capacity of human fibroblasts. Proc Natl Acad Sci USA 1992; 89: 10114.

5. Blackburn EH. Telomeres: structure and synthesis. J Biol Chem 1990; 265: 5919.

6. Blackburn EH, Greider CW, Henderson E, Lee MS, Shampay J, Shippen-Lentz D. Recognition and elongation of telomeres by telomerase. Genome 1989; 31: 553.

7. Blackburn EH. Telomerases. Annu Rev Biochem 1992; 61: 113.

8. Olovnikov AM. A theory of marginotomy. The incomplete copying of template margin in enzymic synthesis of polynucleotides and biological significance of the phenomenon. J Theor Biol 1973; 41: 181.

9. Allsopp RC, Harley CB. Evidence for a critical telomere length in senescent human fibroblasts. Exp Cell Res 1995; 219: 130.

10. Wright WE, Piatyszek MA, Rainey WE, Byrd W, Shay JW. Telomerase activity in human germline and embryonic tissues and cells. Dev Genet 1996; 18: 173.

11. Bonatz G, Klapper W, Barthe A et al. Analysis of telomerase expression and proliferative activity in the different layers of cyclic endometrium. Biochem Biophys Res Commun 1998; 253: 214.

12. Yasumoto S, Kunimura C, Kikuchi K et al. Telomerase activity in normal human epithelial cells. Oncogene 1996; 13: 433.

13. Brien TP, Kallakury BV, Lowry CV et al. Telomerase activity in benign endometrium and endometrial carcinoma. Cancer Res 1997; 57: 2760.

14. Ramirez RD, Wright WE, Shay JW, Taylor RS. Telomerase activity concentrates in mitotically active segments of human hair follicles. J Invest Dermatol 1997; 108: 113

15. Härle-Bachor C, Boukamp P. Telomerase activity in the regenerative basal layer of the epidermis in human skin and in immortal and carcinoma-derived skin keratinocytes. Proc Natl Acad Sci USA 1996; 93: 6476.

16. Hiyama E, Gollahon L, Kataoka T et al. Telomerase activity in human breast tumors. J Natl Cancer Inst 1996; 88: 116.

17. Landberg G, Nielsen NH, Nilsson P et al. Telomerase activity is associated with cell cycle deregulation in human breast cancer. Cancer Res 1997; 57: 549.

18. Yashima K, Milchgrub S, Gollahon LS et al. Telomerase enzyme activity and RNA expression during the multistage pathogenesis of breast carcinoma. Clin Cancer Res 1998; 4: 229.

19. Counter CM, Hirte HW, Bacchetti S, Harley CB. Telomerase activity in human ovarian carcinoma. Proc Natl Acad Sci USA 1994; 91: 2900.

20. Duggan BD, Wan M, Yu MC et al. Detection of ovarian cancer cells: comparison of a telomerase assay and cytologic examination. J Natl Cancer Inst 1998; 90: 238.

21. Tahara H, Kuniyasu H, Yokozaki $\mathrm{H}$ et al. Telomerase activity in preneoplastic and neoplastic gastric and colorectal lesions. Clin Cancer Res 1995; 1: 1245.

22. Tang R, Cheng AJ, Wang JY, Wang TC. Close correlation between telomerase expression and adenomatous polyp progression in multistep colorectal carcinogenesis. Cancer Res 1998; 58: 4052

23. Taylor RS, Ramirez RD, Ogoshi M, Chaffins M, Piatyszek MA, Shay JW. Detection of telomerase activity in malignant and non-malignant skin conditions. J Invest Dermatol 1996; 106: 759.

24. Parris CN, Jezzard S, Silver A et al. Telomerase activity in melanoma and non-melanoma skin cancer. Br J Cancer 1999; 79: 47.

25. Wu A, Ichihashi M, Ueda M. Correlation of the expression of human telomerase subunits with telomerase activity in normal skin and skin tumors. Cancer 1999; 86: 2038.

26. Counter GM, Avilion AA, LeFeuvre GE et al. Telomere shortening associated with chromosome instability is arrested in immortal cells which express telomerase activity. EMBO J 1992; 11: 1921.

27. Holt SE, Wright WE, Shay JW. Regulation of telomerase activity in immortalized cell lines. Mol Cell Biol 1996; 16: 2932.

28. Shay JW. Telomerase in human development and cancer. J Cell Physiol 1997; 173: 266.

29. Kim NW, Piatyszek MA, Prowse KR et al. Specific association of human telomerase activity with immortal cells and cancer. Science 1994; 266: 2011.

30. Shay JW, Bacchetti S. A survey of telomerase activity in human cancer. Eur J Cancer 1997; 33: 787.

31. Rudolph P, Schubert C, Tamm S et al. Telomerase activity in melanocytic lesions: a potential marker of tumor biology. Am J Pathol 2000; 156: 1425.

32. Ramirez RD, D'Atri S, Pagani E et al. Progressive increase in telomerase activity from benign melanocytic conditions to malignant melanoma. Neoplasia 1999; 1: 42.

33. Guttman-Yassky E, Bergman R, Manov L, Sprecher E, Shaefer Y, Kerner H. Human telomerase RNA component expression in Spitz nevi, common melanocytic nevi, and malignant melanomas. J Cutan Pathol 2002; 29: 341.

34. Glaessl A, Bosserhoff A-K, Buettner R, Hohenleutner U, Landthaler M, Stolz W. Increase in telomerase activity during progression of melanocytic cells from melanocytic naevi to malignant melanomas. Arch Dermatol Res 1999; 291: 81.

35. Miracco C, Pacenti L, Santopietro R, Laurini L, Biagioli M, Luzi P. Evaluation of telomerase activity in cutaneous melanocytic proliferations. Hum Pathol 2000; 31: 1018.

36. Tosi P, Miracco C, Santopietro R et al. Possible diagnostic role of telomerase activity evaluation in the differential diagnosis between Spitz naevi and cutaneous malignant melanoma. Br J Dermatol 2000; 142: 1060. 